\title{
Beitrag zur Physiologie der Epidermis mit Bezug auf deren Durchlässigkeit für Licht.
}

Von

Dr. Leopold Freund (Wien).

I.

Es ist bekannt, dass man seit längerer Zeit die Entstehung verschiedener krankhafter Zustände mit der Einwirkung des Sonnenlichtes in Zusammenhang bringt. Speciell glaubte man eine Reihe ron Hautaffectionen auf dieses ätiologische Moment zurückführen zu müssen, und wollte auch gefunden haben, dass die mehr weniger intensiv fortgesetzte Einwirkung des Lichtes auf die Haut einen entschiedenen Einfluss auf den Verlauf der betreffenden Krankheit habe. So beschrieb Hutchinson eine besondere Sommer-Prurigo oder $\mathrm{Sum}$ merEruption, welche an den unbedeckten Hautstellen localisirt ist, Bazin die Hydroa vacciniformis und Unna brachte das Xeroderma pigmentosum mit dem Lichte als ätiologischem Momente in Connex. Es ist bekannt, dass man die Sommersprossen, die Pellagra und das Eczema sowie Erythema solare (Gletscherbrand) ebenfalls aut den Einfluss des Sonnenlichtes zurückführt und das Erythema photoelektricum damit in eine Reihe stellt, welches durch die Einwirkung des elektrischen Lichtes hervorgerufen wird ( $\mathrm{Ch}$ ar cot, Hammer, Widmark, Moeller u. A.).

Schon vor längerer Zeit wurde die Frage aufgeworfen, welchem Bestandtheile des Sonnenlichtes man eigentlich diese 
schädlichen Wirkungen zuzuschreiben habe. Das weisse Licht besteht bekanntlich aus mehreren verschiedenen aber vereinigten Farben und lässt sich unter Umständen (z. B. durch ein Prisma) in die sogenannten sieben Regenbogenfarben zerlegen. Früher meinte man, dass das Rothende des Spectrums vorzüglich thermische, der mittlere Theil desselben, wo es im Gelb, Orange und Grün dem Auge am hellsten erscheint op tische, und das Blauende besonders chemische Eigenschaften besitze. Thatsächlich haben das violette. blaue und selbst grïne Licht auf alle in der Photographie gebrauchten Präparate eine überwiegende Wirkung und man nannte diese Strahlen nach Wollastones Vorgange chemisch-wirksame oder actinische Strahlen. Indessen haben neuere Untersuchungen festgestellt, dass es keine Farbe gibt, welche chemisch absolut unwirksam ist; auch das äusserste Roth wirkt auf Silbersalze, ja auf marche Substanzen äussert das rothe Licht einen stärkeren Einfluss als das violette. Andererseits ist nach neueren Untersuchungen die Wärmewirkung keine specifische Eigenthümlichkeit der schwächer brechbaren rothen und ultrarotben Strablen sondern die wahre Wärmevertheilung ist über alle Spektralabschnitte gleich.

Mehrere Autoren (Widmark, Hammer, Bowles, Mo eller, Finsen u. A.) gaben auf Grund ihrer Experimente an, dass das violette Ende des Spectrums auf die untersuchten Objecte am stärksten einwirke. Sie vindicirten deshalb den sogenannten chemisch-wirksamen Strahlen, und von diesen den jenseits der sichtbaren violetten Strahlen befindlichen ultravioletten Strahlen den Einfluss auf die lebenden Gewebe.

Die Untersuchungen, ïber welche im Nachfolgenden berichtet werden soll, stellten sich die Aufgabe zu prüfen, inwiefern die letzte Annahme berechtigt ist, u. zw. wurde das Augenmerk nicht darauf gerichtet zu untersuchen, ob die ultravioletten Strahlen thatsächlich die hier interessirenden Wirkungen ausuiben, sondern die Frage gestellt, ob die ultravioletten Strahlen denn thatsächlich in der Lage wären eine Wirkung auf die Gebilde in der Tiefe des Corions (Capillargefässe, Nervenenden, Chromatophoren etc.) auszuüben, von welchen sich die verschiedenen krankhaften Zustände der Haut 
ableiten. Da die äussersten ultravioletten Strahlen zum grossen Theile von verschiedenen lichtdurchlässigen Medien z. B. von Glas ${ }^{1}$ ) absorbirt werden. war es interessant zu ermitteln, ob die opaken Oberhautschichten dem Durchtritte dieser Strahlengattung ein wesentliches Hinderniss darbieten. Die Beantwortung dieser Frage hat noch insoferne ein praktisches Interesse, als man jetzt, durch die von Finsen und seinen Mitarbeitern $\mathrm{Bang}$ und Forchhammer mitgetheilten günstigen Resultate der Lichtbehandlung bei Lupus aufmerksam gemacht, der Bestrahlung parasitärer Affectionen mit sog. chemischen Lichtstrahlen lebhaftes Interesse zuwendet. In neuester Zeit ersetzte sogar Strebel die Finsen'sche Behandlung mit elektrischem Bogenlicht durch die Bestrahlung mit dem ultravioletten Lichte des Inductionsfunkens und will damit Bakterienculturen in bedeutend kürzerer Zeit als mit dem Bogenlichte abgetödtet haben. $\left.{ }^{\circ}\right)$ Unter solchen Umständen ist die Kenntniss der Durchlässigkeit der Epidermis für ultraviolette Strahlen von besonderer Wichtigkeit. Denn, wenn die ultravioletten Strahlen thatsächlich so intensiv wirksam sind, und wenn sie in praxi wirklich diese Wirkung in den tieferen Hautschichten, wo der Sitz der krankhaften Veränderungen sich befindet, auszuüben in die Lage kommen, d. h. an ihrem Eintritte zu den letzteren nicht durch die absorbirenden oberflächlichen Gewebe gehindert werden, dann wäre es angezeigt, von der Verwendung der sichtbaren Bestandtheile des Spectrums möglichst abzusehen und nur solche Lichtquellen zu benützen, welche besonders reichlich ultraviolette Strahlen aussenden.

Die diesbezüglichen Untersuchungen sollten daher nicht nur ermitteln, ob dis chemischen und ultravioletten Strahlen in die tieferen Hautschichten eindringen können, um dort ihre Wirkung auszuüben, sie sollten auch im Falle eines positiven Ergebnisses dieser Versuche in möglichst exacter Weise feststellen, welchem Theile des ultravioletten Spectrums diese Eigenthümlichkeit zukommt.

1) Siehe die Abhandlung von Eder und Valenta: Die Spectren farbloser und gefärbter Gläser. Denkschriften der kais. Akad. der Wissenschaft. Math. naturw. Classe, Bd. LXI.

2) Eine Annahme, welche allerdings von Bang bestritten wird. 
II.

Dass die thierischen Gewebe dem auf sie fallenden Lichte, oder zum mindesten gewissen Bestandtheilen desselben, ohne Weiteres den Durchgang gestatten, ist eine seit altersher bekannte Thatsache, und vor Entdeckung der Röntgenstrahlen bediente man sich der Transparenz der Gewebe sogar vielfach zur Exploration der Art und des Sitzes von krankhaften Veränderungen im Innern des menschlichen Körpers. Diese Diaphanität findet bei den verschiedenen Geweben in verschiedenem Grade und in verschiedener Abstufung statt und hängt nicht nur von der Dichtigkeit und chemischen Beschaffenheit sondern vorzüglich von der Gleichartigkeit der Masse der betreffenden Schichte und von deren gleichmässigen Dichtigkeit ab. Die meisten im menschlichen Körper vorkommenden Stoffe sind nicht für alle Farben gleichmässig durchsichtig; sie absorbiren die Strahlen einer oder mehrerer Farben, während sie die Strahlen der anderen Farben durchlassen.

Die Transparenz der Haut lehrt der einfache Versuch, dass beim Vorhalten der Hand vor eine intensive Lichtquelle die Fingerspitzen roth durchschimmern, oder dass man, im Falle man mit geschlossenen Lidern in die Sonne blickt, die Empfindung von rothem Lichte hat. Die täglich geübte Prüfung der Hydrocelen auf ibre Transparenz zeigt ebenfalls in einfacher Weise, dass das Licht die Haut des Scrotums durchdringt. Dessaignes demonstrirte den Durchgang des Lichtes durch die Haut in der Weise, dass er einen Diamanten zum Leuchten brachte, sobald der ihn bedeckende Finger von oben belichtet wurde. Aehnliches fand statt, wenn das Licht durch ein weiss- oder sämisch gegerbtes Fell dringen musste. Einen genaueren Aufschluss über die Natur der die Haut durchdringenden Strablen gab ein Versuch Gadn eff's. Dieser fuillte kleine Röhrchen mit Chlorsilber, schmolz sie zu und brachte sie dann mit Hilfe eines Troiquarts unter die Haut von Hunden und Katzen. Wurden diese Thiere einige Zeit dem Sonnenlichte ausgesetzt, so zeigte sich, dass das Chlorsilber sich geschwärzt hatte, während es bei Controlthieren, die im Dunkeln gehalten worden waren, unverändert blieb. Fin s en bewies den Durcbtritt 
des Lichtes durch die Haut in der Weise, dass er lichtempfindliches Papier hinter das Ohrläppchen brachte und letzteres bestrahlte. Das Papier wurde geschwärzt u. zw. besonders intensiv, wenn durch Compression das Blut aus der Haut verdrängt ward. Gebhard bettete eine Hand so vollständig in Gyps ein, dass nur der Handrücken frei blieb. In die Höhlung der Palma war eine photographische Platte untergebracht. Dann wurde die Hand dem Lichte einer elektrischen Bogenlampe während 20 Minuten ausgesetzt, nachher in der Dunkelkammer von der Platte getrennt und letztere entwickelt. Die Platte erschien geschwärzt, die Contouren der Hand und der Finger traten deutlich hervor: ein Zeichen, dass das Licht durch die Hand hindurchgegangen war. S ol u cha stellte in Bech terews Laboratorium ähnliche Versuche an. Hunden wurden Röhrchen mit Bromsilbergelatinestreifen gefüllt unter die Haut geführt und die Wunde vernäht. Die betreffenden Stellen wurden mit dem Lichte eines elektrischen Projectionsapparates ron 10-20 Amp. Stromstärke und 50-65 Volt bestrahlt. Das Bromsilber erwies sich schon nach $1 / 2$ Minute zersetzt. 'Wenn man die Röhrchen dagegen tief in die Muskelsubstanz der Glutaeen einführte, trat keine Wirkung ein. Beim Ansetzen der Röhrchen hinter das Ohr oder unter die Backe von Patienten trat auch rasche Zersetzung ein, im ersten Falle nach 1/2, im letzten nach 2 Minuten; hinter dem Vorderarme oder in der Faust blieb das Bromsilber selbst nach 15 Minuten unverändert.

Bei obiger Stromstärke dringt also das Licht nur durch die Haut, bei stärkerer dagegen, u. zw. von $25 \mathrm{Amp}$. und 110 Volt, dringt es durcb den ganzen Körper, denn unter diesen Umständen zersetzte sich die Bromsilbergelatineplatte, selbst wenn sie an einem der Applicationsstelle des Lichtes entgegengesetzten Körpertheile placirt wurde: so legte sie Solucha an den Nacken, während er den Vorderhals bestrahlte, oder auf die rechte Körperseite, während das Licht von links kam.

Finsen konnte auch spectroskopisch nachweisen, dass ein durch das comprimirte Ohrläppchen eines Menschen geschickter Lichtstrahl sich noch in alle Farben des Spectrums deutlich zerlegen liess. 
Zum Nachweise der Permeabilität der menschlichen Haut für ultraviolette Strahlen diente Strebel die Eigensehaft der letzteren, fluorescenzfähige Körper zum Leuchten zu bringen. Als Ergebniss dieser Versuche theilte er mit: "Glas saugt ultraviolettes Licht sehr stark auf, Epidermis aber thut dies ganz enorm." Eine ca. $1 \mathrm{Mm}$. dicke Hornplatte löschte das Spectrum schon von Linie 410 ab aus, erschien also sehr wenig durchgängig.

Ein zwischen Quarzplatten eingepresstes menschliches $\mathrm{Ohr}$ absorbirte isolirtes, ultraviolettes concentrirtes Licht vollständig. In einer späteren Mittheilung berichtet $\mathrm{S}$ tr eb el über folgenden Versuch: „Ein Stïck von Fettgewebe frei präparirter Haut wurde zwischen Quarzplättchen geklemmt und unter Ausschluss aller Farbstrahlen mit concentrirtem ultravioletten Lichte, das von Inductionsfunken zwischen Zink und Aluminiumelektroden geliefert wurde, bestrahlt; das Bestrahlungsobject war $140 \mathrm{Cm}$. vom Funken entfernt. Es zeigte sich nun auf dem hinter der Haut aufgestellten Fluorescenzschirm eine schwache, aber sehr deutliche Leuchterscheinung.

Die hier mitgetheilten Versuche zeigen, dass nicht nur die optischen Strahlen, sondern auch chemisch-wirksame Strahlen thierische Gewebe, unter Umständen sogar in beträchtlich dicker Schichte zu passiren vermögen.

\section{III.}

Wie bereits erwähnt, war die gestellte Aufgabe zu untersuchen 1. ob die ultravioletten Strahlen die Epidermis passiren and in die tieferen Hautschichten eindringen können und 2. welchem Theile des ultravioletten Spectrums diese Eigenthümlichkeit zukommt.

Eine exacte Lösung dieser Aufgabe war nur auf spectrographischem Wege möglich, denn wenn auch das Aufleuchten des Fluorescenzschirmes bei Strebel's Versuchen den gleichsam qualitativen $\mathrm{Nachwe}$ is erbrachte, dass ultraviolette Strahlen unter Umständen die Haut zu passiren vermögen, konnte nur die spectrographische Prüfung des durchgedrungenen 
Lichtes genau und mit Sicherheit feststellen, welchem Bestandtheile desselben dieses Verhalten zuzuschreiben ist.

Bekanntlich erfolgt bei den meist üblichen Spectralapparaten die '/erlegung des Lichtes in seine Bestandtheile mit Hilfe eines Glasprismas. Glas besitzt aber wie manche andere durchsichtige Medien die unangenehme Eigenschaft, verschiedene Theile des Spectrums und insbesonders das Ultraviolett $\mathrm{zu}$ absorbiren. Aus diesem Grunde wurde bei den Versuchen von der Verwendung eines Glasprismenspectrographen abgesehen und ein sogenannter Gitterspectrograph benützt. Dieser Apparat erzeugt ein Beugungsspectrum auf die Weise, dass das Licht durch einen Spalt auf einen mit sehr vielen parallel geritzten Linien versehenen Hohlspiegel mit grossem Krümmungsradius (Concavgitter) fällt. Das mit diesem Apparate erhaltene Beugungsspectrum hat nicht nur den Vorzug frei von Absorptionseinflüssen zu sein; es hat vor dem Brechungsspectrum noch das voraus, dass die Ablenkung der Strahlen der Wellenlänge proportional ist, während beim letzteren die stärker brechbaren Strahlen eine verhältnissmässig viel stärkere Ablenkung erfahren, als die schwächer brechbaren, so dass das blaue Ende des Spectrums gegenüber dem rothen ungewöhnlich in die Breite gezogen erscheint.

Hinsichtlich der weiter unten öfters gebrauchten Bezeichnung der Linien im Spectrum sei bemerkt, dass man bei genaueren wissenschaftlichen Untersuchungen die Lage der Linien durch Angabe ihrer Wellenlängen in Ang s trö m'schen Einheiten $(1 A E .=1 / 10000000 \mathrm{Mm}$.) ausdrïckt.

Die im Folgenden beschriebenen Untersuchungen wurden unter Anleitung des Herrn Prof. Eduard Vale nta im photochemischen Laboratorium der k. k. graphischen Lehr- und Versuchsanstalt durchgeführt.

Als Material für diese Untersuchungen, welches ich der Güte der Herren Assistenten Dr. Kreibich und Prof. Lang verdanke, diente frische Epidermis, u. zw. 1. Brandblasen, 2. Blasen von Pemphigus vulgaris. Beide wurden sorgfältig mit der Schere abpräparirt, auf Glaswalzen gerollt und mit diesen in die in einer kleinen Eprouvette aufgefangene Blasen- 
flüssigkeit für den kurzen Transport vom Krankenzimmer ins Laboratorium aufbewahrt.

3. Thiersch'sche Epidermislappen, welche in ähnlicher Weise wie die beiden anderen Präparate in physiologischer Kochsalzlösung aufbewahrt wurden.

Diese verschiedenen Membranen wurden nun sorgfältig auf eine Quarzplatte gebreitet und mit einer ebensolchen Platte gedeckt. Sodann prüfte ich mit starker Lupenvergrösserung, ob das Präparat keine Lücken oder Einrisse besitze. Als diese Untersuchung ein befriedigendes Resultat ergeben hatte, wurden die Quarzplatten vor dem Spalte des Gitterspectrographen befestigt. Als Lichtquelle diente der durch Leydener Flaschen verstärkte Funke eines kräftigen Ruhmkorff'schen Inductoriums, welcher zwischen Elektroden aus einer Legirung von Blei, Zink und Cadmium (Eder'sche Legirung) überschlagen gelassen wurde. Das Spectrum dieser ca. $40 \mathrm{Cm}$. vom Spalte aufgestellten Lichtquelle wurde nun unter Vorschaltung der Präparate, sowie ohne dieselben übereinander photographirt. Die Spaltweite betrug 0.2 Mm., die Belichtungszeit 15 Minuten.

Es ergab sich, dass unter diesen Umständen die Absorption der ultravioletten Lichtstrahlen bei der Cadmiumlinie $\lambda=3250 \mathrm{AE}$ beginnt, $d . h$. dass diese Linie unter den gegebenen Umständen eben noch auf der Platte erkennbar ist, während das Licht der stärker brechbaren Strahlen keine Schwärzung mehr hervorbringt, also absorbirt wird.

Auffallende Unterschiede in der Durchlässigkeit der drei verschiedenen Präparate waren nicht zu constatiren.

In Folge dieser Uebereinstimmung lässt sich mit Sicherheit annehmen, dass von den blauen, violetten und ultravioletten Strahlen diejenigen bis zur Wellenlänge der genannten Cadmiumlinie durch die Epidermis dringen.

Behufs Vergleiches des Verhaltens dieser succulenten, frischen, der normalen Epidermis entsprechenden Präparate mit eingetrockneter wurden folgende Versuche gemacht: Als Materiale dienten Platten aus fast farblosem und etwas gelblich gefärbtem Horn von 0.5 resp. $0.56 \mathrm{Mm}$. Stärke. Die Prüfung derselben wurde unter Zuhilfenahme des beschriebenen Gitterspectrographen und Sonnenlicht durchgeführt. 
Es ergab sich, dass gelb gefärbtes Horn bei einer Spaltbreite von $0.1 \mathrm{Mm}$. und einer Belichtungszeit von 80 Secunden bis zur Frauenhofer'schen Linie $O(\lambda=3441 A E)$ ultraviolettes Licht durchlässt, während unter denselben Umständen bei farblosem Horn die Wirkung der ultravioletten Strahlen bis $\mathrm{Q}(\lambda=3286 A E)$ reicht.

Während also farblose todte Epidermis im Allgemeinen gleiche Absorptionsverhältnisse darbot wie die lebende Oberhaut, erwies sich die Permeabilität gefärbter (pigmentirter) Epidermis gegenüber der ersteren erheblich vermindert.

Bekanntlich hindert das in der Haut circulirende Blut das Eindringen der chemisch wirksamen Lichtstrahlen in erheblicher Weise. Aus diesem Grunde sah sich auch Finsen zur Construction seiner Compressionsapparate veranlasst, mit welchen er die Dauer der Behandlung auch wesentlich abkürzt. Um spectrographisch festzustellen, in welchem Umfange die Absorption der stärker brechbaren Strahlen vom Blute stattfindet, wurden einige Tropfen Blutes aus der Fingerbeere auf eine Quarzplatte gebracht, welche am Rande ringsherum mit einem Streifen Papier ron 0.17 Mm. Dicke abgegrenzt war, und mit einer zweiten Quarzplatte bedeckt. Das Blut füllte den Zwischenraum, welcher zufolge dieser Anordnung 0.17 Mm. betrug, vollkommen aus. Diese Schichte zeigte eine gleichmässig rothe Färbung ohne einen lichten Zwischenraum. Bei Prüfung mit einem kleinen 'Taschenspectroskop zeigte sich das gewöhnliche Absorptionsspectrum des Blutes mit den charakteristischen $\mathrm{Ab}$ sorptionsstreifen zwischen den Frauenhofer'schen Linien D und $\mathrm{E}$ im Grüngelb.

Es wurde nun das Spectrum mit Hilfe eines kleinen Glasspectrographen unter Benützung von Sonnenlicht photographirt. Es ergab sich bei einer Belichtung von 5 Minuten ein Bild des Gesehenen. Die Absorption begann bei $\mathrm{F}^{1 / 2} \mathrm{G}$, und war von da an gegen ultraviolett so gut wie keine Einwirkung auf die photographische Platte $z u$ constatiren.

Die bis jetzt geschilderten Versuche hatten die Epidermis und das Blut zum Gegenstande. Um das Verhalten der lebenden frischen Epidermis, unter welcher Blut circulirt, zu erforschen, wurde ein weiterer Versuch angestellt. 
Ein Frosch wurde curaresirt, dann 2 Zehen eines Hinterfusses an den Rändern des dreieckigen Ausschnittes einer Korkplatte mit Stiften so befestigt, dass die Schwimmhaut über diesen Ausschnitt glatt gespannt hinüberzog. Diese Korkplatte wurde vor dem Spalt des Gitterspectrographen befestigt. Als Lichtquelle diente Sonnenlicht. Die Spaltweite betrug 0.15 Mm., die Belichtungszeit 5 Minuten.

Es ergab sich, dass unter diesen Umständen das Licht von der linie $\mathrm{H}_{1}(\lambda=3968 A E)$ an verlaufend absorbirt wurde.

Wenn man die Dicke der Membran, den Blutreichthum derselben u. s. w. in Betracht zieht, so ist es interessant, dass noch so viel Lichtstrahlen von stärker brechbaren Theilen des Spectrums durchgelassen werden.

Aus allen diesen Versuchen folgt, dass ein beträchtlicher Theil der von verschiedenen Lichtquellen ausgesendeten ultravioletten Strahlen die Epidermis durchdringt und zu den tieferen Hautschichten zu gelangen rermag. Die Menge dieser Lichtstrahlen entspricht ungefähr dem dritten Theil des bisher genauer bekannten ultravioletten Spectrums.

Selbstverständlich werden diese Resultate von der Intensität der Lichtquelle, der Dauer der Einwirkung und der Dicke der bestrahlten Schichte beeinflusst. Bei entsprechender Versuchsanordnung könnte man daher noch günstigere Permeabilitätsverhältnisse der Epidermis constatiren.

Wir wiederholen, dass bei diesen Versuchen die Frage, ob die ultravioletten Strahlen die oben erwähnten krankhaften Zustände der Haut thatsächlich verursachen, ob ihnen die Heilwirkungen der Lichttherapie zuzuschreiben sind und ob letztere wirklich auf der baktericiden oder einer anderen Eigenschaft des kurzwelligen Lichtes beruhen, vollständig in suspenso gelassen wurde; es handelte sich nur darum festzustellen, ob nach den bestehenden Verhältnissen diese Strahlen jene ihnen zu* geschriebenen Wirkungen auszuüben überhaupt in die Lage kommen können.

Es sei bei dieser Gelegenheit nochmals darauf hingewiesen, dass durch neuere photochemische Forschungen nach- 
gewiesen ist, dass nicht nur die kurzwelligen Strahlen, sondern Licht jedweder Wellenlänge resp. Farbe je nach der Natur des von ihm getroffenen Körpers chemische Wirkungen auszuüben vermag. Das Licht wird, wenn es einen Körper trifft und von diesem absorbirt wird, entweder cbemische Arbeit leisten (Oxydations-, Reductionsprocesse), oder es wird in Wärme umgesetzt ohne eine chemische Veränderung hervorzubringen (Absorptionserscheinungen bei lichtecht gefärbten Substanzen), oder es gibt Anlass zu elektrischen Erscheinungen, erregt elektrische Ströme (Photoelektricität), oder beeinflusst das elektrische Leitungsvermögen der isolirten Substanz. Selten verlaufen diese Phänomene vollständig einheitlich, sondern in der Regel treten Complicationen mehrerer derartiger neben einander verlaufender Processe ein. Allerdings wird die Mehrzahl der Körper von den kurzwelligen Strahlen am meisten afficirt, doch ist es bei dem verschiedenen Verhalten der einzelnen thierischen Gewebe und Säftemassen gegen das Licht und bei den variablen Absorptionsspectren dieser Substanzen sehr fraglich, $o b$ man den Finfluss einer einzigen speciellen Lichtgattung auf den menschlichen Organismus einheitlich auffassen kann. Wir erinnern nur an die verschiedenen Absorptionsspectren, welche Blutkörper und Eiweisssubstanzen geben, indem bei ersteren ausser einem Theil der blauen und ultravioletten Strahlen auch langwelliges Licht von bestimmten Bezirken des Spectrums verschluckt wird, während bei Eiweisssubstanzen die kurzwelligen Strahlen die grösste Absorption erleiden.

Jedenfalls sind die animalischen organischen Substanzen, wie aus ihren Absorptionsspectren bervorgeht, sehr reactionsfähig gegen Licht, womit übereinstimmt, dass nach rein empirischen Beobachtungen physiologische Wirkungen auf den Organismus beobachtet wurden.

Bei der Lichttherapie werden heute häufig blaue Flüssigkeitsfilter (Lösungen von Kupferammoniumsulfat) verwendet.

Schaltet man ein solches Lichtfilter in einer für Ultraviolett durchlässigen Wanne (Quarz) in den Gang der Lichtstrahlen, welche rom Funken zwischen Elektroden aus Eder'scher Legirung ausgehen, vor dem Spalte des Gitterspektrographen 
ein und photographirt das Spectrum, so zeigt die entwickelte Platte, dass bei Verwendung einer $10 \mathrm{Mm}$. dicken Schichte von $5 \%$ iger ammoniakalischer Kupfervitriollösung, einer Spaltbreite von $0^{\cdot} 1 \mathrm{Mm}$. und einer Expositionszeit von 3 Minuten das Spectrum bei der Luftlinie $\lambda=3955$ abgeschnittten wird. Es lässt also ein derartiges Lichtfilter unter den geschilderten Umständen blaues und violettes aber so gut wie kein ultraviolettes Licht durch.

Diesen Ergebnissen zufolge wird man in Zukunft bei der Lichttherapie, wenn man auf die Wirkung der ultravioletten Strahlen reflectiren sollte, von der Verwendung dieser blauen Lichtfilter wohl absehen müssen.

Mit Rücksicht auf den durch die hier beschriebenen Versuche festgestellten Antheil des ultravioletten Spectrums, welcher die Epidermis zu durchdringen vermag, liegt es nahe Lichtquellen zu suchen, die bei grosser Intensität vorzüglich Strahlen der brauchbaren Wellenlängen aussenden. Diesbezügliche Arbeiten sind bereits im Gange. Die Ergebnisse derselben sollen später mitgetheilt werden.

Es obliegt mir zum Schlusse, Herrn Director Hofrath Professor Dr. J. M. Eder, mit dessen Einwilligung und Förderung diese Versuche in der k. k. graphischen Lehr- und Versuchs-Anstalt in Wien vorgenommen wurden, sowie Herrn Professor Eduard Valenta, unter dessen Anleitung diese Arbeit ausgeführt wurde, für die Liebenswürdigkeit, mit welcher er mir sein reiches Wissen und Können zur Verfügung stellte, auch an dieser Stelle meinen wärmsten Dank zu sagen. 